\title{
Improving follow-up in hospitalised children
}

\author{
Gary L McPhail, Mathew D Ednick, Matthew C Fenchel, Rhonda VanDyke, \\ Amrita Chima, Raouf S Amin, Michael Seid
}

Division of Pulmonary Medicine, University of Cincinnati, Cincinnati Children's Hospital Medical Center, Cincinnati, Ohio, USA

\section{Correspondence to} Dr Gary L McPhail, Division of Pulmonary Medicine, Cincinnati Children's Hospital Medical Center, ML 2021, 3333 Burnet Avenue, Cincinnati, $\mathrm{OH} 45229$, USA; gary.mcphail@cchmc.org

GLM had full access to all the data in the study and takes responsibility for the integrity of the data and the accuracy of the data analysis.

Accepted 18 January 2010 Published Online First 27 February 2010

\section{ABSTRACT}

Objective To improve the clinic follow-up rate of paediatric inpatients in a tertiary care hospital.

Patients and methods Inpatients who received pulmonary consultations from July 2007 to June 2008 at Cincinnati Children's Hospital Medical Center were eligible for this quality-improvement project. Multiple interventions were introduced to improve follow-up in our subspecialty clinic. A $\chi^{2}$ test for association was used to assess the dependence between the clinic follow-up rate and the type of care coordination intervention. We hypothesised that generalisable care coordination interventions would result in improvements.

Results Two hundred and eleven patients were included. Two interventions were independently associated with improvements in the hospital follow-up rate. When a reminder to follow-up in the pulmonary clinic was inserted into the hospital discharge summary (partial intervention), the clinic follow-up rate improved from $27 \%$ to $55 \%$. When the follow-up appointments were made for the families, with the appointments' noted in the discharge summaries, and automated appointment reminder phone calls were provided (full intervention), the follow-up rate improved further from $55 \%$ to $80 \%$. The full intervention, when compared with no intervention, improved the clinic follow-up rate from $27 \%$ to $80 \%$.

Conclusion Establishing clinic appointments for patients and providing appointment reminders increases the hospital follow-up rate for hospitalised children in outpatient clinics.

\section{INTRODUCTION}

Chronic respiratory diseases are common in childhood and result in significant morbidity and healthcare utilisation. ${ }^{1-8}$ Subspecialty follow-up can improve care in these children. Subspecialty care in asthma increases adherence to asthma guidelines, improves asthma control and decreases hospital admission and readmission rates. ${ }^{9-16}$ Subspecialty care in children with recurrent pneumonias reduces respiratory symptoms, reduces the frequency of recurrent infections and reduces hospital admission rates. ${ }^{17-20}$ Subspecialty care in children with obstructive sleep apnoea syndrome (OSAS) is important to monitor for metabolic and neurocognitive sequelae, to assess for the recurrence of sleep apnoea after surgical interventions and to optimise adherence to positive pressure therapies. ${ }^{5} 2122$

Previous studies on follow-up rates in children with chronic respiratory diseases have focused on primary care follow-up rates in children with asthma after emergency department visits. ${ }^{23-26}$ Primary care follow-up rates in this setting are low, and range from 19 to $46 \% .^{23-26}$ Care coordination improves these follow-up rates. ${ }^{24-26}$ Smith et al improved the emergency department follow-up rate in children from $19 \%$ to $36 \%$ with intensive asthma coaching and monetary incentives. ${ }^{24}$ Baren et al improved the follow-up rate from $42 \%$ to $65 \%$ with free asthma medications, free travel vouchers and preset follow-up clinic appointments. ${ }^{25}$ These interventions by Smith and Baren were effective, but are cost prohibitive for most clinical settings. Zorc et al improved the emergency department follow-up rate in children from $46 \%$ to $64 \%$ with one low cost, generalisable intervention. ${ }^{26}$ They established follow-up appointments for patients before they were discharged from the emergency department. ${ }^{26}$ This intervention efficiently removed barriers to patient follow-up. Families did not need to call to establish follow-up appointments. They were not forced to navigate the phone systems of the primary care physicians, and they were not hindered by the lack of outpatient appointment availability. Further, the importance of follow-up visits was reinforced by the efforts of the study staff to establish these appointments.

The goal of our quality improvement study was to take the approach of Zorc et al to the paediatric inpatient setting. We tested low-cost, generalisable care coordination interventions to remove barriers to hospital follow-up, in an attempt to improve the follow-up rate in our subspecialty clinic.

\section{PATIENTS AND METHODS Patients}

This was a local quality-improvement project and per local standard of practice, informed consent and Institutional Review Board approval were not obtained. Patients were included in this project if they received inpatient pulmonary consultations between 1 July 2007 and 30 June 2008 at Cincinnati Children's Hospital Medical Center. These patients were chosen for inclusion, since a prior analysis revealed that their follow-up rates in the outpatient clinic after hospital discharge were low. Patients were excluded from the project if the consultations were only for a procedure (flexible bronchoscopy) or only for postoperative respiratory management. Patients were excluded from the project if they died or were transferred to an inpatient pulmonary service during hospitalisation, if they were already an established patient in the pulmonary outpatient clinic, or if they had any other local subspecialty respiratory follow-up already established.

\section{Interventions}

Interventions were introduced sequentially. Interventions that did not improve follow-up were not 
continued. Interventions that improved clinic follow-up were expanded. During the first month of the study, direct mail reminders were sent to families. The direct mail reminders stressed the importance of clinic follow-up and encouraged families to call for appointments. This intervention resulted in a low follow-up rate (only $3 / 27=11 \%$ of patients followed-up) and was subsequently stopped. During the second month of the study, patients received either a follow-up reminder in person (delivered during hospitalisation) or no follow-up reminder based on a random number table. The group that received an inperson reminder to follow-up trended to a higher follow-up rate when compared with the group that received no reminder. Fifty per cent $(5 / 10)$ of patients in the in-person reminder group followed up in clinic compared with $0 \%(0 / 6)$ of patients in the no reminder group $(p=0.09$, Fisher exact test). This approach was modified and expanded. In subsequent months, reminders to follow-up were inserted into the hospital discharge summary instead of being given in person. In subsequent months, patients received no mention in the hospital discharge summary that follow-up was needed in the pulmonary clinic (no intervention), received a mention in the hospital discharge summary that follow-up was needed in the pulmonary clinic (partial intervention) or received a mention in the hospital discharge summary that pulmonary follow-up was needed, had an appointment established for them by an administrative assistant, had the clinic appointment date and time noted in the discharge summary prior to hospital discharge, and received an automated reminder phone call within $72 \mathrm{~h}$ of the clinic appointment (full intervention). Patients were not randomised to these final interventions, but a combination of interventions was used during each month. During this study, a hospital-wide service provided the automated reminder phone calls to all families within $72 \mathrm{~h}$ of a scheduled outpatient appointment. This was not specific to our intervention process but was clearly an important part of the intervention bundle.

Clinic appointments were established by two administrative assistants in the Pulmonary Division. The administrative assistants were notified via email from the inpatient pulmonary consultation team when a follow-up appointment was needed. The administrative assistants contacted the families in the hospital by phone when possible. The administrative assistants established the next available pulmonary clinic appointment that was amenable to the families' scheduling preferences, including time, day and clinic location. The average time spent by the administrative assistants in this process per patient was $10 \mathrm{~min}$. Costs of the administrative assistants' time were estimated at $\$ 3.78 /$ per patient based on the average salary and fringe benefits for administrative assistants in the Pulmonary Division. The cost of the automated phone system could not be estimated, as this was installed at the hospital level, and the pricing of similar commercial products is proprietary information and not advertised by the distributing companies.

\section{Statistical methods}

The Fisher exact test was used after the second month of the study to compare the follow-up rates of patients who received in-person reminders for follow-up with those who did not receive in-person reminders. In subsequent months, the three levels of discharge summary care coordination were used, and interventions were defined as (1) no intervention=no pulmonary follow-up mention in the discharge summary; (2) partial intervention=mention of the need for pulmonary follow-up in the discharge summary only; or (3) full intervention=mention of the need for pulmonary follow-up, the clinic appointment made by staff, the date and time of appointment documented in the discharge summary and a reminder phone call provided to families within $72 \mathrm{~h}$ of the scheduled clinic visit. Pulmonary clinic follow-up within 90 days of hospital discharge (yes or no) was obtained for each patient.

A $\chi^{2}$ test was used to assess the overall dependence between follow-up rate and type of care coordination intervention. The Cochran-Armitage trend test was used to assess trends in the degree of intervention (none, partial and full) and probability of successful follow-up in clinic after discharge. ${ }^{27}$ The degree and strength of association between intervention and follow-up was measured using the Somer D. Subsequently, ORs, RR ratios and differences in proportions were computed (comparing full intervention versus no intervention, partial intervention versus no intervention and full intervention vs partial intervention), along with 95\% CIs for the ORs and RR ratios, and 95\% asymptotic CIs for the differences in proportions calculated. This provided further assessment of whether or not the odds and probabilities of clinic follow-ups were greater for each level of intervention.

\section{RESULTS}

Two hundred and eleven patients were included in the analysis. The primary discharge diagnoses for these patients were asthma (31\%), community-acquired and/or complicated pneumonia $(27 \%)$, OSAS $(18 \%)$, aspiration pneumonia (13\%) and other $(11 \%)$. The clinic follow-up rate after hospital discharge was strongly dependent on the care coordination intervention $\left(p<0.0001, \chi^{2}\right.$ test for independence). Further, the probability of returning for follow-up increased as the extent of intervention increased (Cochran-Armitage test for trend, $\mathrm{p}<0.0001$; Somer D measure of association: $0.35,95 \%$ CI $(0.25,0.44))$. Patients who received follow-up reminders in their hospital discharge summaries (partial intervention) were more likely to come to the clinic within 90 days than patients who received no reminders (see table 1). Patients who received follow-up reminders in their hospital discharge summaries had the appointments established by administrative assistants, had the dates and times of the appointments noted in their discharge summaries and received automated appointment reminder phone calls (full intervention), and had higher probabilities and higher odds of coming to clinic within 90 days than patients who received only discharge summary reminders (partial intervention; see table 1). The clinic no-show rate for patients who had appointments established prior to hospital discharge was $20 \%(11 / 54)$, consistent with our overall outpatient clinic no show rate of $17 \%$ for the 1 July 2007 to 30 June 2008 study period.

\section{DISCUSSION}

The hospital follow-up rate in our study improved from $27 \%$ to $80 \%$ with simple care coordination interventions. Establishing follow-up appointments and providing reminder phone calls to families led to this improvement. Our baseline follow-up rate was low, but our baseline rate was no different from the followup rates noted by Smith, Baren and Zorc. ${ }^{24-26}$ Low follow-up rates are common in clinical settings, but improvements in clinic attendance can be achieved with focused interventions. ${ }^{24-2628-31}$ Engelstad et al improved follow-up in a group of low-income, minority women by using community health advisors to schedule patient appointments, make reminder phone calls and provide patient education. ${ }^{28}$ Szilagyi et al concluded, in both a prospective trial and meta analysis, that appointment reminder systems 
Table 1 Probabilities and ORs of clinic follow-up after hospitalisation: comparing different levels of inpatient care coordination interventions

\begin{tabular}{llll}
\hline Intervention levels & Follow-up rates & RR (95\% CI)* & OR (95\% CI)† \\
\hline $\begin{array}{l}\text { Full intervention }(n=54) \text { versus no } \\
\text { intervention }(n=91)\end{array}$ & $80 \%$ versus $27 \%$ & 2.9 (2.02 to 4.15) & 10.3 (4.6 to 23.1) \\
$\begin{array}{l}\text { Partial intervention }(n=66) \text { versus no } \\
\text { intervention }(n=91)\end{array}$ & $55 \%$ versus $27 \%$ & 1.99 (1.33 to 2.96) & 3.2 (1.6 to 6.2$)$ \\
$\begin{array}{l}\text { Full intervention }(n=54) \text { versus partial } \\
\text { intervention }(n=66)\end{array}$ & $80 \%$ versus 55\% & $1.5(1.1$ to 1.9$)$ & 3.3 (1.4 to 7.4$)$ \\
\hline
\end{tabular}

* Ratio of the probability of follow-up in the Pulmonary Clinic within 90 days of hospital discharge in the exposed group versus the nonexposed group, where degree of exposure refers to the different levels of care coordination interventions.

tOdds of following-up in the Pulmonary Clinic within 90 days of hospital discharge when comparing different levels of care coordination interventions.

improved the rates of preventive care in children and reduced healthcare disparities. ${ }^{29} 30$ In a meta-analysis of randomised trials, Macharia et al found that patient education, direct mail reminders and telephone reminders improved clinic attendance. ${ }^{31}$ Direct mail reminders were not successful in our study. Direct mail reminders may not have been successful in our study, since only a limited number of patients received them $(n=27)$. It is also possible that there were significant inaccuracies in our hospital database for patients' home addresses.

Discharge planning, care coordination and specialty follow-up in hospitalised patients with chronic diseases improve patient outcomes. ${ }^{32-34}$ Broyles et al randomised very-low-birth-weight infants to comprehensive care after hospital discharge and found fewer life-threatening illnesses and fewer intensive care unit admissions in the comprehensive care group. ${ }^{32}$ Phillips et al concluded that comprehensive discharge planning was associated with lower readmission rates and greater improvements in quality of life in adults with congestive heart failure. ${ }^{33}$ Naylor et al showed that discharge planning and frequent follow-up were associated with lower rates of rehospitalisation and lower healthcare costs in elderly patients with chronic illnesses. ${ }^{34}$

Our study adds to the current literature. Children with chronic diseases are a vulnerable population targeted by the Institute of Medicine as a priority for transforming healthcare by improving healthcare delivery. ${ }^{35}$ Our study used simple care coordination interventions to improve the hospital follow-up rates in this population. We established patient appointments and utilised hospital discharge summaries to deliver patient reminders. Automated appointment reminder phone calls were provided by a hospital-wide computer-based system. These interventions can be implemented in most clinical settings and would be expected to improve clinic follow-up rates. ${ }^{26} 28-31$

Our quality-improvement study was limited. This project involved a single paediatric centre. Patients were not randomised to the final care coordination interventions. The possibility for bias exists within this study design. Appointment reminder phone calls were provided by a hospital-wide service. The relative contributions of establishing patient appointments versus providing appointment reminder phone calls could not be determined. Future studies are needed to define the balance between resources required in care coordination and improvements in patient follow-up rates.

\section{CONCLUSIONS}

Care coordination, including arranged clinic appointments and reminder phone calls, increases hospital follow-up rates for paediatric patients with chronic diseases. These generalisable care coordination interventions improved our clinic follow-up rate from $27 \%$ (no intervention) to $80 \%$ (full intervention). Simple interventions have the potential to improve healthcare delivery for all hospitalised patients with chronic diseases, at little cost to the healthcare system.

Acknowledgements We are indebted to U Kotagal and G Kaminski for their wisdom in helping design this improvement project through the Intermediate Improvement Science Series at Cincinnati Children's Hospital Medical Center. We would like to thank L Guerrant and E Clayton, the administrative assistants who contacted the families and scheduled the follow-up clinic visits during this study. Without their help, this project would not have been successful.

Funding This study was funded internally. There was no sponsor involvement in the design of this study or in the writing of this manuscript for publication.

\section{Competing interests None.}

Provenance and peer review Not commissioned; externally peer reviewed.

\section{REFERENCES}

1. Moorman JE, Rudd RA, Johnson CA, et al. National surveillance for asthma-United States, 1980-2004. MMWR CDC Surveill Summ 2007:56:1-54.

2. Martinez FD. Trends in asthma prevalence, admission rates, and asthma deaths. Respir Care 2008;53:561-5.

3. Cropper JA, Frank TL, Frank PI, et al. Respiratory illness and healthcare utilization in children. Eur Respir J 2001;17:892-7.

4. Amin R, Somers VK, McConnell K, et al. Activity-adjusted 24-hour ambulatory blood pressure and cardiac remodeling in children with sleep disordered breathing. Hypertension 2008;51:84-91.

5. Gozal D, Capdevila OS, Kheirandish-Gozal L. Metabolic alterations and systemic inflammation in obstructive sleep apnea among nonobese and obese prepubertal children. Am J Respir Crit Care Med 2008;177:1142-9.

6. Zhao 0, Sherrill DL, Goodwin JL, et al. Association between sleep disordered breathing and behavior in school-aged children: the Tucson Children's Assessment of Sleep Apnea Study. Open Epidemiol J 2008;1:1-9.

7. Castro-Rodríguez JA, Holberg CJ, Wright AL, et al. Association of radiologically ascertained pneumonia before age 3 yr with asthmalike symptoms and pulmonary function during childhood: a prospective study. Am J Respir Crit Care Med 1999;159:1891-7.

8. Owayed AF, Campbell DM, Wang EE. Underlying causes of recurrent pneumonia in children. Arch Pediatr Adolesc Med 2000;154:190-4.

9. National Heart, Lung, and Blood Institute. Expert Panel Report 3 (EPR3): guidelines for the diagnosis and management of Asthma. Bethesda (MD): National Heart, Lung, and Blood Institute, 2007:1-440.

10. Najada A, Abu-Hasan M, Weinberger M. Outcome of asthma in children and adolescents at a specialty-based care program. Ann Allergy Asthma Immunol 2001:87:335-43.

11. Bucknall CE, Robertson C, Moran F, et al. Differences in hospital asthma management. Lancet 1988;1:748-50.

12. Mayo PH, Richman J, Harris HW. Results of a program to reduce admissions for adult asthma. Ann Intern Med 1990;112:864-71.

13. Kelly CS, Morrow AL, Shukts J, et al. Outcomes evaluation of a comprehensive intervention program for asthmatic children enrolled in Medicaid. Pediatrics 2000;105:1029-35

14. Castro M, Zimmermann NA, Crocker $\mathrm{S}$, et al. Asthma intervention program prevents readmissions in high healthcare users. Am J Respir Crit Care Med 2003;168:1095-9.

15. Diette GB, Skinner EA, Nguyen $T T H$, et al. Comparison of quality of care by specialist and generalist physicians as usual source of asthma care for children. Pediatrics 2001;108:432-7

16. Guarnaccia S, Lombarda A, Gaffurini A, et al. Application and implementation of the GINA asthma guidelines by specialist and primary care physicians: a longitudinal follow-up study on 264 children. Prim Care Resp J 2007;16:357-62.

17. Boesch RP, Daines C, Willging JP, et al. Advances in the diagnosis and management of chronic pulmonary aspiration in children. Eur Respir $J$ 2006;28:847-61 
18. Gerber ME, Gaugler MD, Meyer CM, et al. Chronic aspiration in children. When are bilateral submandibular gland excision and parotid duct ligation indicated? Arch Otolaryngol Head Neck Surg 1996;122:1368-71.

19. Mattioli G, Sacco O, Repetto P, et al. Necessity for surgery in children with gastrooesophageal reflux and supraoesophageal symptoms. Eur J Pediatr Surg 2004:14:7-13.

20. Busse PJ, Razvi S, Cunningham-Rundles C. Efficacy of intravenous immunoglobulin in the prevention of pneumonia in patients with common variable immunodeficiency. J Allergy Clin Immunol 2002:109:1001-4.

21. Marcus CL, Rosen G, Ward SL, et al. Adherence to and effectiveness of positive airway pressure therapy in children with obstructive sleep apnea. Pediatrics 2006;117:e442-51.

22. Amin R, Anthony L, Somers V, et al. Growth velocity predicts recurrence of sleepdisordered breathing 1 year after adenotonsillectomy. Am J Respir Crit Care Med 2008;177:654-9.

23. Zorc JJ, Scarfone RJ, Li Y. Predictors of primary care follow-up after a pediatric emergency visit for asthma. J Asthma 2005;42:571-6.

24. Smith SR, Jaffe DM, Fisher EB Jr, et al. Improving follow-up for children with asthma after an acute emergency department visit. J Pediatr 2004;145:772-7.

25. Baren JM, Boudreaux ED, Brenner BE, et al. Randomized controlled trial of emergency department interventions to improve primary care follow-up for patients with acute asthma. Chest 2006;129:257-65.

26. Zorc JJ, Scarfone RJ, Li Y, et al. Scheduled follow-up after a pediatric emergency department visit for asthma: a randomized trial. Pediatrics 2003;111:495-502.
27. Agresti A. Categorical data analysis. 2nd edn. New York: Wiley-Interscience, 2002.

28. Engelstad LP, Stewart S, Otero-Sabogal R, et al. The effectiveness of a community outreach intervention to improve follow-up among underserved women at highest risk for cervical cancer. Prev Med 2005;41:741-8.

29. Szilagyi PG, Schaffer S, Shone L, et al. Reducing geographic, racial, and ethnic disparities in childhood immunization rates by using reminder/recall interventions in urban primary care practices. Pediatrics 2002;110:e58.

30. Szilagyi PG, Bordley C, Vann JC, et al. Effect of patient reminder/recall interventions on immunization rates: a review. JAMA 2000;284:1820-7.

31. Macharia WM, Leon G, Rowe BH, et al. An overview of interventions to improve compliance with appointment keeping for medical services. JAMA 1992; 267:1813-17.

32. Broyles RS, Tyson JE, Heyne ET, et al. Comprehensive follow-up care and lifethreatening illnesses among high-risk infants: a randomized controlled trial. JAMA 2000;284:2070-6.

33. Phillips C0, Wright SM, Kern DE, et al. Comprehensive discharge planning with postdischarge support for older patients with congestive heart failure: a metaanalysis. JAMA 2004;291:1358-67.

34. Naylor MD, Brooten D, Campbell R, et al. Comprehensive discharge planning and home follow-up of hospitalized elders: a randomized clinical trial. JAMA 1999;281:613-20.

35. Institute of Medicine. Priority Areas for National Action: transforming Health Care Quality. The National Academies Press 2003. 